\title{
RAPID LOSS OF VISION WITH SWELLING OF THE OPTIC DISC
}

Acute papillitis, cranial arteritis, and atherosclerotic ischaemic optic neuropathy are conditions in which rapid loss of vision may be accompanied by swelling of the optic disc. They often present similar clinical pictures in the early stages and it is important to distinguish between them as there is a considerable difference in their prognosis and treatment.

Acute Papillitis is characterized by loss of vision over 2 or 3 days and occurs most commonly in the third to fifth decades, although it may present at any age between 5 and 60 years. It is usually unilateral in adults, although the second eye may be involved at a later date. The loss of vision is typically, although not invariably, accompanied by pain on moving the eye and tenderness over the tendon of the superior rectus muscle.

Oedema of the optic nerve-head occurs in acute papillitis. The hyperaemia of the disc is accompanied by only moderate dilatation of the retinal veins adjacent to it. Retinal haemorrhages are not common and if present are few in number. Retinal arterioles are normal (Fig. 1).

Central or paracentral scotomata are the usual field defects found in this condition. The prognosis for full recovery of vision is good, particularly in patients under 45 years of age (96 per cent. recovery rate), although only about 75 to 80 per cent. of patients above this age recover a visual acuity of $6 / 9$ or better.

Treatment in acute papillitis is generally not indicated; ACTH appears to accelerate the rate of recovery, although probably not the final visual result.

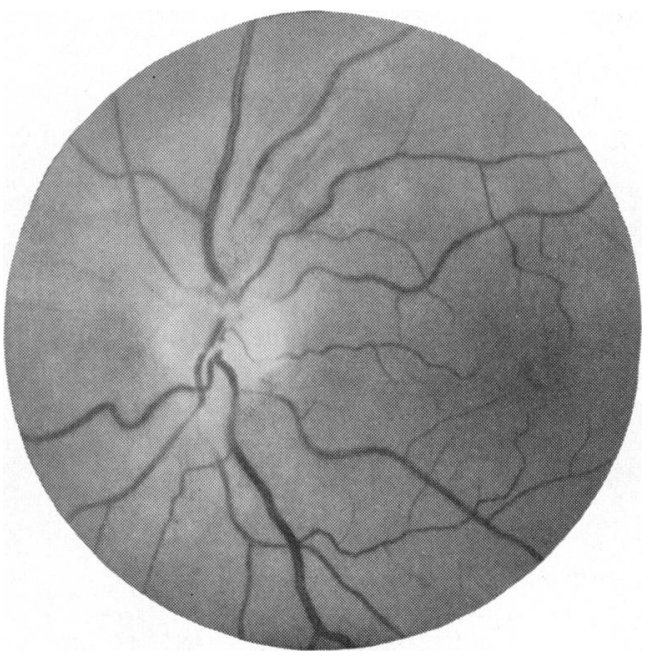

FIG. 1.-Acute papillitis.

Cranial Arteritis occurs most commonly in patients over 70 and is rare in those under 60 years of age. Although it usually affects one eye first, in the absence of treatment it is a bilateral disease.

Headache is usually a prominent feature of cranial arteritis but it may occasionally be absent. The pain may involve the occipital and facial as well as the temporal region. The superficial temporal arteries are usually tender and palpably thickened, often with little or no pulsation, in contrast to the thickened pulsating vessels in atherosclerosis. Tender nodules may be present along these arteries. Pain often precedes the loss of vision by some weeks and may even have disappeared by the time visual symptoms start. Loss of vision is sudden, often progressing to no perception of light in a few hours. If perception of light is retained, it is often limited to a small island of peripheral vision. There is no ocular pain. 
Cranial arteritis may involve the central artery of the retina producing the typical appearance of occlusion of this artery. More often the arteritic process involves the small branches of the ophthalmic artery within the optic nerve, producing an ischaemic swelling of the optic disc. The disc appears swollen but has a greyish pallor with retinal haemorrhages and soft exudates restricted to the margins of the disc and peripapillary region. The retinal arterioles show little narrowing.

The erythrocyte sedimentation rate is usually grossly raised, but cases have been described in which it was normal.

Once vision has been affected, the prognosis in cranial arteritis is very poor. Immediate treatment with high doses of systemic steroids is essential to prevent involvement of the second eye.

Atherosclerotic Ischaemic Optic Neuropathy is a condition in which there is atherosclerotic and/or hypertensive occlusive disease of the small nutrient branches of the ophthalmic artery in the anterior part of the optic nerve. It occurs in patients over the age of 50 years and cases of ischaemic swelling of the optic disc occurring under the age of $\mathbf{7 0}$ are more commonly atherosclerotic than arteritic in nature.

Atherosclerotic ischaemic optic neuropathy is usually a painless condition, although ocular pain similar to that occurring in optic neuritis has occasionally been reported. Loss of vision is sudden in onset but is usually less profound than in cranial arteritis.

The optic nerve-head appears swollen and ischaemic and the retinal vessels show atherosclerotic and/or hypertensive changes and may be very attenuated (Fig. 2).

Various types of visual field defect may be present and, although central scotomata may occur, nerve fibre bundle defects, irregular peripheral constrictions, and altitudinal defects (usually of the lower field) are more commonly found.

The erythrocyte sedimentation rate in atherosclerotic ischaemic optic neuropathy is usually normal, in contrast to the usually grossly raised rate found in cases of cranial arteritis.

The prognosis for recovery of vision is poor and, although little or no improvement can be expected, deterioration to complete

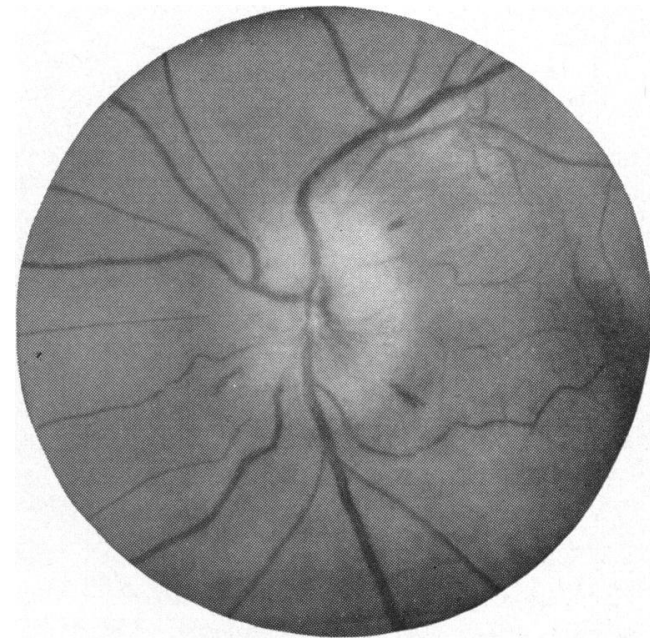

FIG. 2.-Ischaemic optic neuropathy. blindness is rare.

There is no effective treatment for this disease once it is established, although measures to increase the blood flow in the ophthalmic artery may be beneficial in the early stages. These include a retrobulbar injection of Priscol, ocular massage, and systemic Diamox.

Illustrations:

INSTITUTE OF OPHTHALMOLOGY, UNIVERSITY OF LONDON.
BRIAN MARTIN, THE LONDON HosPITAL, LONDON, E.1. 